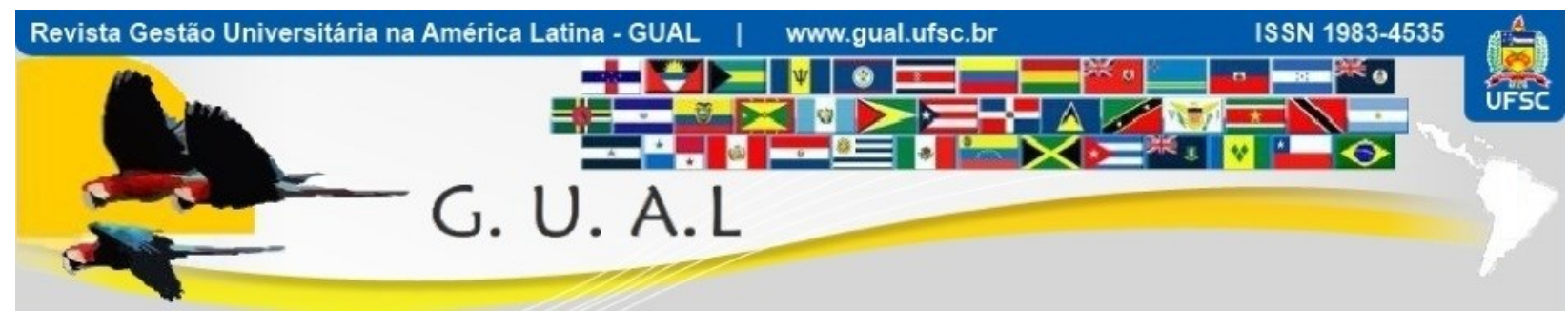

DOI: http://dx.doi.org/10.5007/1983-4535.2016v9n1p97

\title{
CONTRIBUIÇÃO DAS COMUNIDADES DE PRÁTICA PARA O PROCESSO DE AUTOAVALIAÇÃO EM UMA INSTITUIÇÃO DE EDUCAÇÃO SUPERIOR DO SEGMENTO PRIVADO
}

\section{CONTRIBUTION OF COMMUNITIES OF PRACTICE FOR SELF ASSESSMENT PROCESS ON A PRIVATE SEGMENT INSTITUTION OF HIGHER EDUCATION}

Gustavo Tomaz Buchele, Doutorando Universidade Federal de Santa Catarina - UFSC gustavotb.adm@,gmail.com

João Artur de Souza, Doutor Universidade Federal de Santa Catarina - UFSC jartur@gmail.com

Marilda Todescat, Doutora Universidade Federal de Santa Catarina - UFSC marildat@gmail.com

Maristela Denise Coelho, Mestranda Universidade Federal de Santa Catarina - UFSC $\underline{\text { maricoelho adm@hotmail.com }}$

Mileide Marlete Ferreira Leal Sabino, Doutoranda Universidade Federal de Santa Catarina - UFSC mileide.sabino@gmail.com

Thiago Henrique Almino Francisco, Doutorando Universidade do Extremo Sul Catarinense - UNACSA

Recebido em 22/abril/2015 proftf@gmail.com

Aprovado em 10/novembro/2015

Sistema de Avaliação: Double Blind Review

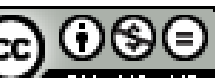

Esta obra está sob uma Licença Creative Commons Atribuição-Uso. 


\title{
RESUMO
}

$\mathrm{Na}$ educação superior, a gestão do conhecimento ainda é um conceito que carece de um esclarecimento singular para que as práticas emergentes possam se diluir nos modelos institucionais existentes. Nesse sentido, as comunidades de prática (CoPs) surgem como instrumento de relevância substancial à construção de uma cultura de avaliação. Desse modo, o objetivo deste artigo é o de identificar em que medida as CoPs podem colaborar com o processo de autoavaliação em uma instituição de educação superior. Para a consolidação da pesquisa, buscou-se ainda a caracterização da comunidade de prática que é objeto do estudo, a identificação das lacunas do processo de autoavaliação da instituição estudada, a discussão do processo que ocorre por meio das CoPs e a apresentação das propostas e ações de gestão do conhecimento que surgiram a partir da atividade da comunidade de prática que se constituiu na instituição em análise. No que tange à metodologia, trata-se de um estudo predominantemente qualitativo, descritivo, bibliográfico, documental, por meio da análise de conteúdo e estudo de caso. Os resultados oriundos da pesquisa, por sua vez, demonstraram que as comunidades de práticas colaboram e permitem uma difusão do conhecimento de forma sistemática e inovadora.

Palavras-Chave: Gestão do Conhecimento. Comunidades de Prática. Ensino Superior.

\begin{abstract}
In higher education, knowledge management is still a concept that lacks a natural explanation for emerging practices may dilute the existing institutional models. In this sense, communities of practice (CoPs) appear as substantial relevance instrument to building a culture of evaluation. Thus, the purpose of this article is to identify the extent to which CoPs can collaborate with the self-assessment process in a higher education institution. In order to consolidate the effort even sought to characterize the community of practice that is the object of study, the identification of gaps in the self-assessment process of the study institution, the discussion of the process that occurs through the CoPs and the submission of tenders and management actions of knowledge that emerged from the practice of community activity that constituted the institution in question. With respect to methodology, it is a predominantly qualitative, descriptive study, bibliographic, documentary, through content analysis and case study. The results arising from the research, in turn, showed that communities of practice collaborate and enable dissemination of knowledge in a systematic and innovative way.
\end{abstract}

Keywords: Knowledge Management. Communities of Practice. Higher Education. 


\section{INTRODUÇÃO}

A gestão do conhecimento busca o desenvolvimento de práticas que permitem a consolidação da gestão organizacional, voltada à criação de valor e conhecimento útil nos processos. Como o campo é multidisciplinar, uma série de ferramentas, instrumentos e técnicas determinam a convergência de ações nas diversas, e difusas, esferas organizacionais, permitindo e orientando a criação de valor por meio das pessoas, das tecnologias, dos processos, das lideranças organizacionais e, principalmente, da cultura organizacional.

Independentemente do modelo de organização, a busca pelo conhecimento crítico promove a qualificação das pessoas envolvidas no processo, gerando valor, e permite que tecnologias sejam convergentes aos objetivos organizacionais. Por meio dos esforços de gestão do conhecimento é possível determinar a construção de ativos intangíveis e de capital intelectual, os quais se configuram em ativos para novos modelos de negócio. $\mathrm{O}$ conhecimento é entendido como algo fundamental quando utilizado, levando à proposição de diversos modelos teóricos afins aos objetivos em função da necessidade das organizações.

Dentre as diversas práticas que surgem para fortalecer a dinâmica da gestão do conhecimento organizacional, as comunidades de prática (CoPs) aparecem como sendo um instrumento de relevância substancial na construção de uma cultura de utilização do conhecimento, já que favorece a interação entre os detentores do conhecimento tácito e cria padrões que minimizam o empobrecimento do processo de explicitação de conhecimento.

$\mathrm{Na}$ educação superior, sobretudo nas instituições que são caracterizadas por ABMES (2014), percebe-se que a gestão do conhecimento ainda é conceito que carece de um esclarecimento para que as práticas emergentes possam se diluir nesses modelos institucionais. Porém, àquelas já alinhadas às práticas de gestão do conhecimento percebem resultados importantes dessa utilização, dentre eles a melhoria significativa em processos, o desenvolvimento de uma cultura voltada ao conhecimento e a constituição de uma dinâmica de inovação, a qual permite um posicionamento competitivo no segmento de atuação.

Baseado nesse aspecto, o artigo se propõe a apresentar as principais contribuições produzidas por uma comunidade de prática que se envolveu com o processo de auto avaliação em uma instituição de educação superior privada. No caminho para a consolidação do estudo, buscam-se também a caracterização da comunidade de prática que é objeto do estudo, a identificação das lacunas do processo de auto avaliação da instituição estudada. 


\section{FUNDAMENTAÇÃO TEÓRICA}

Neste capítulo é apresentado um arcabouço teórico dos principais conceitos Gestão do Conhecimento, Comunidades de Prática e a dinâmica do processo de auto avaliação do ensino superior brasileiro, servindo de base à análise dos resultados desta pesquisa.

\subsection{GESTÃO DO CONHECIMENTO: DEFINIÇÕES E MODELOS}

O conhecimento, na atual economia, é o mais importante fator de produção, de agregação de valor, de produtividade e de crescimento econômico (DOS SANTOS, 2005; BRINKLEY, 2006). Nesse sentido, o conhecimento é conceituado como uma "mistura fluida de experiência condensada, valores, informação contextual e insight experimentado, a qual proporciona uma estrutura para avaliação e incorporação de novas experiências e informações" (DAVENPORT; PRUSAK, 1999, p. 6). No entanto, para a evolução do conhecimento, torna-se necessário diferenciá-lo de dados e de informações. Assim, um dado é um número ou palavra que não apresenta nenhum sentido ou contexto, enquanto a informação se caracteriza a partir das relações entre os dados em um determinado contexto e seus resultados poderão ter uma implicação no futuro. Já o conhecimento, por sua vez, apresenta padrões com a tendência de autocontextualização (URIARTE JR, 2008).

Partindo-se do entendimento de Sveiby (1998) de que o conhecimento possui um caráter iminentemente tácito e individual com foco na ação a partir de normas e com mudanças constantes, na prática tal conhecimento é explicitado através das habilidades e experiências do indivíduo. Sendo assim, é possível perceber a existência de dois tipos de conhecimento: conhecimento tácito e conhecimento explícito. Segundo Uriarte Jr. (2008), o conhecimento tácito é pessoal, sendo armazenado no cérebro das pessoas e de difícil formalização, armazenamento e articulação. O conhecimento explícito é aquele que pode ser codificado e armazenado em documentos e em outras formas que não sejam no cérebro humano, além de poder ser transmitido ou compartilhado de forma sistemática com mais facilidade.

Os dois tipos de conhecimentos interagem de quatro maneiras, formando uma espiral de criação do conhecimento, e podem ser representados em quatro quadrantes. Esse conhecimento à medida que é explicitado tem capacidade de ser compartilhado a partir do indivíduo para o conhecimento de grupo, organizacional, até fazer parte do conhecimento da sociedade. 


\section{CONTRIBUIÇÃO DAS COMUNIDADES DE PRÁTICA PARA O PROCESSO DE AUTOAVALIAÇÃO EM \\ UMA INSTITUIÇÃO DE EDUCAÇÃO SUPERIOR DO SEGMENTO PRIVADO \\ DOI: http://dx.doi.org/10.5007/1983-4535.2016v9n1p97}

Para Uriarte Jr. (2008), estas interações entre os conhecimentos tácito e explícito são: socialização (tácito individual para tácito em grupo); externalização (tácito para explícito); combinação (explícito de forma fragmentada para explícito de forma sistemática); e internalização (explícito para tácito). Jyoti et al. (2011) enfatizam que a Gestão do Conhecimento compreende a administração do conhecimento individual e organizacional através da gestão do conhecimento organizacional atual e da criação de novos conhecimentos. Assim, define-se Gestão do Conhecimento como a conversão do conhecimento tácito em conhecimento explícito, compartilhando-o na organização por meio dos processos de identificação, aquisição, distribuição e manutenção do conhecimento (URIARTE Jr., 2008).

A Gestão do Conhecimento, por oportuno, torna-se um fator indispensável por meio da aplicação de três direcionadores: criar, construir e manter uma vantagem competitiva através da utilização de práticas de conhecimento e colaboração; conhecimento usado para reduzir a complexidade no processo de inovação; integração de conhecimentos internos e externosà organização, tornando-os acessíveis e disponíveis (DU PLESSIS, 2007). Nesse sentido, as capacidades de inovação contínuas de uma organização estão intimamente associadas com seus sistemas e processos de gestão do conhecimento (CHAPMAN; HYLAND, 2004).

Assim, existem diversos modelos relativos tanto à implementação quanto à avaliação do conhecimento como vantagem competitiva para as organizações, sendo os principais modelos de implementação: modelo de Wiig (1993) em que o conhecimento deve ser organizado em um dado contexto para ser útil; modelo de von Krogh e Roos (1995) que distingue o conhecimento individual do social, modelo I-Space de Boisot (1998), o qual é baseado no conceito chave de que uma boa informação se difere de um ativo físico, modelo Complex Adaptative System (ICAS) que consiste em agentes independentes que interagem um com outros localmente combinando comportamentos que irão gerar um fenômeno adaptativo complexo (DAKLIR, 2005), e modelo de Choo (1998) que descreve a Gestão do Conhecimento a partir da formação de sentido, criação de conhecimento e tomada de decisão. Já os modelos de avaliação levantados foram: modelo de Apostolou et al. (1998), representação gráfica dos ativos de conhecimento e os objetos de conhecimento, e o modelo de Handzic et al. (2008), framework por conhecimento, processos, facilitadores, drivers, resultados e contingências. 


\section{CONTRIBUIÇÃO DAS COMUNIDADES DE PRÁTICA PARA O PROCESSO DE AUTOAVALIAÇÃO EM \\ UMA INSTITUIÇÃO DE EDUCAÇÃO SUPERIOR DO SEGMENTO PRIVADO \\ DOI: http://dx.doi.org/10.5007/1983-4535.2016v9n1p97}

Dubois e Wilkerson (2008) apresentam a importância das pessoas na Gestão do Conhecimento, pois a aprendizagem organizacional requer a transmissão do entendimento de um indivíduo ou disposição para mudança de comportamento organizacional. A cultura, o conteúdo, os processos e as tecnologias, sozinhos, não gerenciam o conhecimento.

Quando o conhecimento é capturado e codificado, ele deve ser compartilhado e disseminado por toda a organização (DALKIR, 2005). Nesse sentido, à medida que o aprendizado é um evento predominantemente social, para que se utilize o conhecimento eficaz, existem as comunidades de prática para auxiliar em tal processo (COHEN; PRUZAK, 2001). As Comunidades de Prática, por sua vez, são tema abordado no próximo capítulo.

\subsection{COMUNIDADES DE PRÁTICA: CARACTERÍSTICAS E APRENDIZAGEM}

As comunidades de prática são grupos de pessoas que possuem interesses pessoais e profissionais em comum, que compartilham, participam e estabelecem amizades. Tais grupos produzem conhecimento por meio de suas interações, criando uma memória grupal para a organização (DALKIR, 2005). Segundo Wenger e Snyder (2000), as CoPs possuem o objetivo de desenvolver as competências dos integrantes, além de criar e compartilhar conhecimentos.

Segundo a APO (2010), as CoPs são uma ferramenta importante de aplicação em processos de Gestão do Conhecimento. Algumas características das CoPs são enfatizadas por Wenger (1998) e Dalkir (2005), como o objetivo comum, em geral, pela melhoria da profissão dos membros; participação com base na confiança, interesse e profissionalismo e, por fim, a existência de um espaço de trabalho virtual para discussões. Não obstante, ainda, podem ser destacadas por três características: prática (define as práticas de trabalho propriamente ditas de maneira especialista); comunidade (grupo de pessoas que compartilham conhecimentos de interesse comum); e domínio (conhecido como 'empresa comum', ou seja, é a missão de atingir os objetivos em comum) (WENGER, 1998; WENGER; SNYDER, 2000).

O desenvolvimento e manutenção das CoPs depende de sete princípios: projeto para a evolução; abertura de diálogo entre perspectivas internas e externas; diferentes níveis de participação; desenvolver espaços comunitários públicos e privados; foco no valor; combinar familiaridade e excitação; criar um ritmo para a comunidade. (WENGER et al., 2002).

Todavia, a aprendizagem nas CoPs depende de diferentes níveis de interação. Assim sendo, Wenger (1998) destaca três diferentes maneiras de captura dos modos de participação 
a fim de promover um processo mútuo para coordenar perspectivas, interpretações, e ações para atingir os objetivos maiores, quais sejam: engajamento, imaginação e alinhamento. Ainda, quanto à área educacional, a aprendizagem não é apenas um meio para um fim, fazendo com que a perspectiva de CoPs se torne relevante. Nas instituições de ensino, a alteração da teoria da aprendizagem é uma transformação mais profunda, podendo levar mais tempo.

\subsection{DINÂMICA DA AUTO AVALIAÇÃO NO ENSINO SUPERIOR BRASILEIRO}

O crescimento na oferta do ensino superior no Brasil, a partir da década de 1980, a partir das reformas do sistema de ensino superior dos países industrializados e latinoamericanos (INEP, 2007), é essencial à profissionalização e para atendimento das exigências de mercado.

Segundo Stallivieri (2006), a educação de nível superior brasileira passou por cinco fases, podendo ser definidas do seguinte modo: (a) a partir de 1920 - surgimento da primeira universidade no País inicia uma nova era, ainda que seu caráter seja voltado menos à investigação/pesquisa que ao ensino; (b) de 1930 a 1964: da Revolução Industrial tardia experimentada à implementação do governo militar, mais 20 universidades federais foram criadas; (c) 1968: fase das reformas educacionais de âmbito universitário, buscando a indissociabilidade entre ensino, pesquisa e extensão; (d) 1970: a década marcou o desenvolvimento de cursos de pós-graduação; (e) 1990: década em que inicia a vigência da CF de 1988 e a regulação e otimização do sistema de avaliação do ensino superior.

Assim, em 1993 surge o Programa de Avaliação Institucional das Universidades Brasileiras (PAIUB), originário de experiências anteriores, tendo como objetivo a auto avaliação, por meio da adesão voluntária das universidades e a avaliação externa das mesmas. Sua importância está na conquista da legitimidade da avaliação como cultura e mecanismo para a qualidade do ensino perante as universidades, o governo e a sociedade. (INEP, 2007)

Inevitavelmente, com a retirada do apoio do Ministério da Educação (MEC) ao Programa, o mesmo se tornou ferramenta optativa às universidades, focando a auto avaliação institucional, somente. Ao passo, demais instrumentos foram aprimorados a fim de suprir a necessidade de supervisionar e gerir o ensino nas IES, como o Exame Nacional de Cursos (ENC), a Análise das Condições de Ensino (ACE) e o Questionário sobre Condições Socioeconômicas dos alunos, reunindo informações dos resultados das instituições e 
classificando-as em um ranking que incentivara a competitividade entre elas. (RIBEIRO, 2000)

Entrementes, de acordo com Barreyro e Rothen (2006) a percepção voltada aos resultados e às análises quantitativas, em geral, deixou à margem a real função de um sistema de avaliação das IES, o de diagnosticar a área a fim de conduzir políticas públicas de educação.

Nesse viés, a Lei no 9.394/1996 - Lei de Diretrizes e Bases da Educação (LDB) - traz em seu escopo o processo de avaliação e as responsabilidades e atribuições dos agentes e esferas estatais. Em um processo contínuo de aperfeiçoamento, ao ser sancionada a Lei $n^{\circ}$ 10.172/2001, criou-se o Plano Nacional de Educação (PNE) avolumando prerrogativas da LDB e do art. 214 da CF/88 para consolidar o Sistema Nacional de Avaliação.

Nada insólita a articulação entre os níveis de governo para a efetivação do PNE, visando a cooperação do sistema federal e dos sistemas estaduais de educação e vice-versa, a fim de construir de um sistema nacional de avaliação. Destarte, a sanção da Lei $n^{o}$ 10.861/2004 instituiu o Sistema Nacional de Avaliação do Ensino Superior, composto por três modalidades de análise: avaliação das instituições de ensino superior (AVALIES), avaliação dos cursos de graduação (ACG) e avaliação de desempenho acadêmico dos estudantes (ENADE).

Pontualmente, em relação às modalidades de análise para avaliação estabelecida no SINAES, há de se contextualizar suas órbitas de atuação e funcionalidade dentro do sistema:

1. Avaliação das Instituições de Ensino Superior (AVALIES): compreende dois momentos de análise, quais sejam, auto avaliação da IES e avaliação externa. A primeira, permeia o espaço de auto reconhecimento da funcionalidade organizacional, propiciando a autocrítica que resulta no aprimoramento das atividades. Para tanto, são analisadas as 10 dimensões propostas pelo SINAES: (a) Missão e Desenvolvimento Institucional; (b) A Política para o Ensino, a Pesquisa, a Pós-graduação e a Extensão; (c) Responsabilidade Social; (d) Comunicação com a Sociedade; (e) Política de Pessoal; (f) Organização e Gestão Institucional; (g) Infraestrutura Física; (h) Planejamento e Auto avaliação; (i) Política de Atendimento aos Alunos Egressos e, (j) Sustentabilidade Financeira. Para sua realização, a IES deve formar sua Comissão Própria de Avaliação (CPA), contando com a participação de representantes de todos os segmentos universitários e da sociedade civil, devendo elaborar o Relatório de Auto avaliação, a ser enviado à Comissão Externa de Avaliação (CEA). 
Evidentemente, a CEA representa o início da segunda etapa da AVALIES, na qual a percepção externa acerca da IES, com base no estudo documental e visitação técnica, permite mapear fragilidades e fortalezas.

2. Avaliação de Cursos de Graduação (ACG): consiste em procedimento utilizado pelo MEC para o reconhecimento ou renovação de reconhecimento dos cursos de graduação, representando uma medida necessária para a emissão de diplomas e legitimidade do curso.

3. Exame Nacional de Avaliação de Desempenho de Estudantes (ENADE): visa mensurar o desempenho dos estudantes com relação aos conteúdos programáticos dos cursos de graduação, o desenvolvimento de competências ao aprofundamento da formação profissional e o nível de atualização dos estudantes. Assim, aplica-se o exame aos acadêmicos ingressantes e egressos dos cursos de graduação, resultando em uma classificação.

Segundo Stallivieri (2006), o processo de avaliação não diz respeito somente a correção de possíveis falhas do processo, pois a auto avaliação institucional tem por consequência a racionalização de recursos orçamentários, a eficiência de políticas internas e o desenvolvimento técnico-acadêmico, capacitando o aparato administrativo para a gestão do conhecimento.

Entrementes, impraticável se torna a construção de um sistema de avaliação em nível nacional em que os paradigmas não estejam alinhados. Logo, os três subsistemas do SINAES oferecem uma visão panorâmica e real da avaliação da educação superior brasileira, mensurando-a numérica (caráter quantitativo) e simbolicamente (caráter qualitativo).

Pode-se inferir que o SINAES busca por meio da intersecção de suas partes e de modo interdisciplinar, por sua vez, promover o cumprimento dos aspectos regulatórios legais no processo de credenciamento, renovação e descredenciamento junto ao MEC, associando-o ao atendimento das diretrizes sociais inerentes à educação. (RIBEIRO, 2000)

Sendo assim, a seguir apresentam-se os procedimentos metodológicos utilizados na consecução desse estudo. Ademais, abordar-se-ão na sequência os resultados de análise do caso observado e as lacunas observadas no processo de auto avaliação das IES estudada.

\section{METODOLOGIA}

O paradigma dominante da pesquisa científica, de acordo com Creswell (2010), vem passando por uma transformação em função das diversas reflexões que se constituem e que tem como base a desconstrução dos padrões hegemônicos do que é considerado ciência. 
Desse modo, o estudo que se constitui a partir dessa pesquisa busca se enquadrar em um escopo que é considerado, dentro das bases de Sommerman (2006), um campo interdisciplinar.

A partir dessa base epistemológica, o artigo se pauta na visão de mundo interpretativista, utilizando um paradigma destacado por Morgan (1980), considerando a realidade construída pelos agentes de um determinado contexto como base paradigmática para a interpretação do fenômeno, tendo os eventos de uma comunidade de prática, no contexto do artigo.

A investigação caracteriza-se, ainda, uma pesquisa qualitativa, de acordo com Taylor e Bogdan (1984), já que busca o entendimento de eventos que fazem sentido para um determinado corpo social. Embora possa existir um caráter subjetivo, a investigação constrói fundamentos sólidos que são entendidos como concretos em um determinado contexto.

Traçando-se uma perspectiva de interdependência com outros paradigmas, a pesquisa considera os apontamentos de Souza, Fialho e Otani (2007) e Zapelini e Zapelini (2007), configurando-se como bibliográfica e documental. A utilização de materiais bibliográficos e documentais que fundamentam o arcabouço da investigação ajudam a construir a base teórica.

Ainda com base nas definições dos autores, a pesquisa ainda se configura como sendo descritiva e explicativa, já que busca a descrição processual de um evento e explica suas interlocuções com diversas circunstâncias que fomentam uma realidade epistêmica. Importa destacar que a investigação também se apropriou da entrevista não-estruturada, baseando-se em um roteiro que buscou criar um ambiente significativo a partir da participação de um agente diretamente envolvido, conforme Miles e Huberman (1994).

A investigação se constitui como sendo um estudo de caso, a qual se encontra sustentada pelos pressupostos de Yin (2001), a fim de um entendimento sistematizado da realidade empírica, com uma visão de mundo indutiva que fortalece o aspecto integrativo da investigação.

\section{APRESENTAÇÃO DOS RESULTADOS: LACUNAS DO PROCESSO DE AUTO AVALIAÇÃO DA IES OBJETO DE ESTUDO}

A auto avaliação surge como um processo substancial para a educação superior a partir da instituição do Sistema Nacional de Avaliação da Educação Superior (SINAES) e ganha espaço como sendo um processo de apoio à gestão institucional desde sua concepção. 


\section{CONTRIBUIÇÃO DAS COMUNIDADES DE PRÁTICA PARA O PROCESSO DE AUTOAVALIAÇÃO EM \\ UMA INSTITUIÇÃO DE EDUCAÇÃO SUPERIOR DO SEGMENTO PRIVADO \\ DOI: http://dx.doi.org/10.5007/1983-4535.2016v9n1p97}

De acordo com Augusto e Balzan (2007), percebe-se que o processo se esforça para ganhar legitimidade no contexto institucional, sobretudo no segmento privado.

Ao depender do Plano de Desenvolvimento Institucional (PDI), como determina Brasil (2006), a auto avaliação deveria fortalecer o planejamento institucional e fomentar a discussão sobre o processo. Porém, no segmento privado isso não ocorre em função do conflito de interesses no processo de auto avaliação, confrontando práticas que são a práxis desse modelo institucional e amplia uma série de lacunas que o processo possui.

A instituição que compõe o estudo, "IES A", tem na auto avaliação um mecanismo de geração de conhecimento útil para os processos institucionais. Até o ano de 2011, quando era gerenciada por uma equipe multidisciplinar, a instituição buscava o desenvolvimento do conhecimento crítico da instituição, fortalecendo uma cultura de utilização do conhecimento. Esse aspecto favoreceu a dinâmica gerencial da instituição e a tornou uma das referências regionais nos indicadores de qualidade, conforme Portaria Normativa 40/2007.

Durante o período de 2008 a 2011, onde uma decisão estratégica acabou substituindo todo o corpo diretivo, a instituição teve na auto avaliação seu principal mecanismo de discussão sobre estratégias acadêmicas e administrativas. Tal aspecto se deu em função da decisão de implantação de mecanismos de gestão do conhecimento em processos críticos da instituição.

Durante três anos, na "IES A", a comunidade acadêmica reconheceu a auto avaliação como sendo um mecanismo de diálogo e discussão sobre a dinâmica administrativa, pedagógica, estratégica e acadêmica da instituição. A CPA, que durante o período de dois anos se transformou em uma comunidade de prática envolvida com o processo de avaliação institucional, promoveu uma série de estudos que congregavam os objetivos de promover a avaliação como o principal instrumento de definição e validação das estratégias da instituição e de atuar como uma comissão que buscava criar uma visão de mundo específica.

Resgatando-se um trabalho realizado a partir de 2009, podem-se elencar algumas lacunas. A primeira se alinha ao discurso produzido no trabalho de Garcia (2006), já que a vivência dos envolvidos no processo, sobretudo a dos coordenadores de curso, é um aspecto relevante para a qualidade dos resultados produzidos. Na composição da comissão, fator determinado como obrigatório por INEP (2009), identificou-se que a experiência dos envolvidos era substancialmente diferenciada, já que o coordenador, com apenas seis meses de experiência na área, absorvia a função de nortear o trabalho de um membro que possuía 
mais de 10 anos de vivência técnica. Isso se deu, principalmente, em função da disponibilidade, já que o coordenador era contratado apenas e especificamente para orientar esse processo.

Já a segunda lacuna se envolve com o trabalho Covac (2011), considerando-se que o arcabouço legal que orienta a regulação da educação superior é complexo e deve ser observado pela auto avaliação para que o processo possa atender as dimensões que são propostas pelo SINAES. Além disso, há também um aspecto significativo relacionado à dinâmica multidisciplinar do processo, causando diferentes visões sobre sua importância e eficácia.

A principal lacuna do processo está baseada nas orientações do INEP (2009), quando apresenta a estrutura e a dinâmica sugerida para a operacionalização da auto avaliação. Em função da abrangência das etapas, as dificuldades em construir o projeto, escolher a metodologia em função das diversas visões de mundo que se apresentavam, de construir e aplicar os instrumentos, de comunicar os resultados e, sobretudo, de realizar a meta avaliação, orientaram a reflexão da CPA sobre a importância da qualificação de suas atividades.

Foi por isso que, amparados nas premissas e definições da gestão do conhecimento organizacional, buscou-se a constituição de uma comunidade de prática para o estudo das lacunas que surgiram, permitindo e orientando o uso de conhecimento útil para o processo e validando as atividades, instrumentos, regulamentos e dinâmicas norteadoras que se constituíram ao longo do desenvolvimento de auto avaliação. A escolha pela utilização da CoP se justificou pela vivência de dois, dos cinco membros, no contexto acadêmico da gestão do conhecimento, e se deu ao longo de dois, dos cinco anos em que a CPA se constituiu.

\subsection{A COP COMO INSTRUMENTO DE CONSTRUÇÃO DE CONHECIMENTO: INSTRUMENTOS E ENCAMINHAMENTOS PRODUZIDOS}

As comunidades de prática são mecanismos alinhados ao conceito de gestão do conhecimento e que fortalece o processo de sistematização e de explicitação de conhecimento.

O termo, cunhado por Wenger (1998), define como as organizações informais vão se relacionar no contexto da troca de experiências, incitando a criação de um modelo e de métodos de compartilhamento de conhecimento em um determinado contexto. Nesse caso, para a organização em estudo, é possível perceber que a CoP criada é um mecanismo de prática social que visa a modificação da práxis instituída em um determinado segmento da instituição. 


\section{CONTRIBUIÇÃO DAS COMUNIDADES DE PRÁTICA PARA O PROCESSO DE AUTOAVALIAÇÃO EM \\ UMA INSTITUIÇÃO DE EDUCAÇÃO SUPERIOR DO SEGMENTO PRIVADO \\ DOI: http://dx.doi.org/10.5007/1983-4535.2016v9n1p97}

Como anteriormente definida, a $\mathrm{CoP}$ liga grupo de pessoas com interesses comuns para que compartilhem suas experiências e fortaleçam a estrutura de conhecimento útil da organização. Isso faz com que a criatividade possa incentivar novas ideias e a tomada de decisão, articulando e criando um ciclo virtuoso do conhecimento. (SCHOMMER, 2005)

\subsubsection{Bases para a Constituição da CoP}

A multidisciplinaridade busca o desenvolvimento de dinâmicas pluralistas que se voltam à construção de uma nova epistemologia, ou então o fortalecimento da discussão sobre epistemologias já existentes que visam o desenvolvimento de uma visão de mundo coerente com o objeto em estudo. É sob esse pressuposto que Sommerman (2006) salienta que os estudos multidisciplinares devem se constituir, permitindo que todos os membros que estudam uma determinada comunidade científica possam se envolver substancialmente no estudo.Constituída a partir de 2008 e contando com a participação de sete indivíduos de diferentes formações e diversas experiências no âmbito da educação superior, a CoP criada buscou, propositalmente, a participação de todos os envolvidos na CPA da instituição em estudo, aproveitando a experiência desse grupo de indivíduos no processo de avaliação.

Desse modo, a $\mathrm{CoP}$ se constituiu por meio das ações dos seguintes agentes: a administrador com experiência em Gestão do Conhecimento, psicóloga, responsável pela Direção Acadêmica da instituição, Pedagoga, representante dos docentes, analista de sistemas, analista das atividades de avaliação, acadêmico do curso de graduação em Administração e, por fim, representante da Sociedade Civil Organizada.

Outro ponto substancial que se destaca foi o fato de que a $\mathrm{CoP}$ escolheu um recorte metodológico para sua atividade no momento em que se estabelece para discutir a dinâmica do SINAES a partir de suas etapas, criando métodos para operacionalizar a auto avaliação em observância aos critérios já instituídos pelos documentos norteadores do processo. Desse modo, seguindo as diretrizes de INEP 2004, a CoP se consolidou para discutir métodos e alinhar as atividades de preparação, desenvolvimento e consolidação das atividades de auto avaliação.

Os demais participantes contribuíram com suas visões enviesadas para cada uma das representações, já que todas tinham função substancial e participação validada por ato legal na comissão própria de avaliação da instituição. Dessa forma, ao escolher a dinâmica de atividade alinhada às etapas do processo avaliativo, a CoP passou a se debruçar em discussões 
que promoveram alguns resultados ao longo de sua constituição, os quais seguem descritos a seguir.

\subsubsection{As atividades na $1^{\text {a }}$ etapa: $O$ envolvimento da CoP na preparação da auto avaliação}

Ao delimitar as atividades a partir das etapas do processo de auto avaliação, a CoP que se constituiu na instituição buscou amparo em diversos materiais para entender o funcionamento da dinâmica de uma comissão de avaliação interna em uma instituição de educação superior. As orientações do SINAES, promovidas pelo INEP (2009) e por Augusto e Balzan (2007) foram substanciais na validação da ideia de legitimar a CoP, considerada uma comunidade informal de discussão sobre o conhecimento, como uma comissão formal em função do dinamismo de suas atividades e do envolvimento de cada um dos membros. Além de se constituir como um diferencial competitivo para a auto avaliação, isso confirma o que destaca o trabalho de Silva e Alfredo (2011), já que a CPA deve contar com dedicação substancial de seus membros para que desenvolva com plenitude suas atividades.

A partir da orientação do coordenador dos trabalhos, a CoP criou um instrumento para validar as principais atividades que deveriam ser desenvolvidas por cada um dos membros da CPA, consolidando um formulário de descrição de funções para cada um dos envolvidos com a auto avaliação. Isso se justifica, sobretudo, pelo caráter temporal que é previsto para a atividade de cada comissão, que em geral não é maior do que dois anos e depende de um processo eleitoral que pode ser previsto em regulamento. Esse instrumento permitiu que o conhecimento sobre as principais atividades de cada um dos envolvidos pudesse ser compartilhado, fortalecendo o dinamismo de cada uma das representações envolvidas.

Com a criação desse instrumento, a $\mathrm{CoP}$ se reuniu para definir as linhas gerais do projeto de auto avaliação, buscando inserir as demandas de todos os segmentos no processo avaliativo. Isso trouxe a primeira percepção de que a CoP, definitivamente, assumia o papel da CPA e fortaleceu a coesão do grupo já que a ideia inicial era a de que os encontros seriam apenas informais, porém os envolvidos na CoP, de comum acordo, entenderam que assumir as atividades da CPA seria um importante desafio e uma valorosa contribuição à instituição. Desse modo, a diretriz nessa etapa estava relacionada com as atividades de busca, efetuadas por cada uma das representações envolvidas na CoP, pelas principais demandas que eram consideradas interdependentes para a avaliação da instituição. 
O resultado foi a construção de um projeto que atendia ao disposto no Decreto No 5.773, de 09 de maio de 2006, já que o documento permitiu que a auto avaliação pudesse se constituir em um alinhamento com os direcionamentos legais, especialmente no sentido de atender a avaliação da qualidade, a regulação e a supervisão, quando isso era necessário. $\mathrm{O}$ projeto permitiu que, entre outros aspectos, fossem observados os indicadores de qualidade da educação superior que acabavam de ser instituídos pelas orientações da Portaria Normativa No 40, de 13 de dezembro de 2007. O projeto foi criado de maneira inovadora, em relação aos demais que eram produzidos ao longo de outras instituições, já que era segmentado por dimensões que levavam em consideração as demandas dos segmentos envolvidos.

Com o projeto definido e as linhas de atividade bem alinhadas, a CoP se debruçou na construção das dinâmicas de sensibilização, onde a contribuição de cada um dos segmentos foi importante para a proposição de um método alinhado com a linguagem de todos os envolvidos.

Isso culminou no desenvolvimento de um evento pioneiro na instituição, denominado de " $1{ }^{\circ}$ Fórum de Reflexão Social da IES A", que ocorreu, de acordo com o projeto do evento, entre os dias 22 a 26 de setembro de 2008 e tinha o objetivo de discutir, no âmbito de cada um dos segmentos sociais envolvidos com a instituição, a dinâmica do processo de auto avaliação. Na estrutura do evento, diversas dinâmicas que foram operacionalizadas estiveram sob a responsabilidade dos diversos segmentos representados na comissão, e permitiu que a "IES A" obtivesse o maior percentual de participação em um processo avaliativo desde sua fundação, já que mais de $70 \%$ de todas as representações colaboraram com o desenvolvimento do processo, o qual é detalhado no próximo segmento do trabalho.

\subsubsection{As atividades da $2^{\mathrm{a}}$ etapa: As contribuições da CoP no desenvolvimento da auto avaliação}

$\mathrm{Na}$ linha do que discutem Wenger (1998) e Dalkir (2005), a CoP que se constituiu ao longo do processo promoveu o desenvolvimento sistemático de um ambiente informal e inseriu a cultura de compartilhamento de conhecimento em um contexto complexo. Isso gerou autonomia nos membros participantes, havendo um envolvimento dinâmico no processo de socialização das principais estruturas que compunham o projeto de avaliação.

Um primeiro movimento idealizado a partir da atividade das $\mathrm{CoP}$ foi dividir o grupo em segmentos e orientar a apresentação do projeto às respectivas representatividades. Além de permitir a escolha da melhor linguagem e de uma abordagem customizada e diretamente 
relacionada com as expectativas de cada segmento da comunidade acadêmica, esse envolvimento permitiu que o projeto ganhasse a credibilidade e o envolvimento de toda a comunidade acadêmica, fortalecendo o processo de auto avaliação da instituição. Esse processo ocorreu por meio de um evento formal, no qual a $\mathrm{CoP}$ se apresentou formalmente como a CPA da instituição e destacou os principais desafios que envolveram cada um dos participantes.

Após essa discussão, concluía-se o processo de socialização do projeto e iniciava-se a discussão sobre a dinâmica dos instrumentos. De acordo com a Ata da $4^{\text {a }}$ reunião de 2008 , identificou-se o envolvimento direto do Analista de Sistemas e do Analista das Atividades de Avaliação no processo de construção e validação dos instrumentos. O documento não destaca os procedimentos estatísticos utilizados, mas o que se percebe por meio da análise do relatório final apresentado em 2009, é a utilização de um procedimento complexo de análise de dados, o que trouxe ainda mais credibilidade para o processo.

Isso ficou claro na apresentação dos dados, os quais foram tabulados com o auxílio da utilização de um sistema informatizado construído exclusivamente para essa finalidade. Vale destacar que o responsável pela atividade da CoP, o Administrador com expediência em gestão do conhecimento (AGC), entrevistado nesse trabalho, destaca que foi uma das principais contribuições da $\mathrm{CoP}$ que se perdeu logo após o final das atividades dessa comunidade, em função de uma nova alteração significativa na gestão devido a questões políticas e gerenciais.

Na conclusão do processo, de acordo com a $5^{\mathrm{a}}$ Ata de reunião de 2008 , percebe-se que há um significativo sentimento de realização que permeou as reuniões, contando com a participação do principal gestor da instituição, o qual convidou uma série de personalidades políticas para acompanhar a apresentação prévia do relatório final.

O documento apresentou um consistente trabalho realizado, principalmente em função dos dados apresentados e dos encaminhamentos sugeridos, os quais renderam uma série de prêmios de reconhecimento aos participantes da CoP, inclusive em termos pecuniários. Após essa apresentação inicial, alguns dados foram ajustados, em termos estatísticos para atender a linguagem e o entendimento de cada segmento representado. 


\subsubsection{As atividades da $3^{\text {a }}$ etapa: As reflexões da CoP na consolidação da auto avaliação}

No momento em que se reuniu com o Conselho Superior da "IES A" a CoP, de acordo com a $7^{\text {a }}$ Ata de Reunião de 2008, consolidou-se como um mecanismo importante para a instituição. Os dados do relatório enviado ao Ministério da Educação em 2009 comprovaram o envolvimento de cada um dos membros no processo e permitiu que a cultura de avaliação pudesse se fortalecer no contexto da instituição e ser reconhecida pelos gestores. Com a participação de $100 \%$ de todo o staff da instituição, foi possível identificar que realmente a avaliação passava a fazer parte da cultura da comunidade acadêmica.

Os resultados apresentados ao Conselho Superior, os quais estão destacados no Relatório Final de Auto Avaliação do ano de 2008, submetido ao MEC em 2009, mostrou que um estudo sistemático e dinâmico foi desenvolvido e a identidade institucional da "IES A" foi colocada em discussão para a busca de melhorias e para a busca da construção de uma imagem de qualidade. O AGC, coordenador do processo, destaca como um dos momentos de maior satisfação, já que o trabalho de todos era reconhecido em âmbito institucional e permitiu que diversos participantes pudessem se aprofundar no conceito e na carreira da avaliação.

Ao final da apresentação, a Ata destaca que outro instrumento inovador foi apresentado. No relato do AGC, percebe-se que a meta avaliação foi proposta a partir das instruções do Program Evaluation Standards: How to Assess Evaluations of Educational Programs, do Joint Committe (1994), buscando fortalecer a consistência do processo. Além disso, os encaminhamentos propostos foram incluídos no plano de trabalho da gestão, como mostra o Relatório Inicial do PDI de 2009, sendo que o presidente da CPA passaria a compor um assento no Conselho Superior da instituição, consolidando a credibilidade proposta pela avaliação.

Apesar do conjunto de sugestões e da dinâmica que a avaliação proporcionou à IES, condições externas afetaram a atividade da $\mathrm{CoP}$ e os trabalhos foram encerrados em função do desligamento de três dos membros. Pelo relato do ACG, a CPA permaneceu, porém sem a mesma representatividade em função de um desgaste político interno à instituição. 


\section{CONSIDERAÇÕES FINAIS}

As análises deste estudo foram pautadas no arcabouço legal criado para regular o Sistema Nacional de Avaliação da Educação Superior (SINAES), almejando-se alinhar mecanismos de otimização da educação superior às práticas de gestão de conhecimento.

Nessa perspectiva, se por um lado o sistema em estudo apresenta uma amplitude maior em relação a políticas anteriores de avaliação, por outro, a interação dos resultados obtidos através da avaliação de seus componentes não satisfaz a concepção sistemática a qual se propõe o SINAES. Especificamente, cada uma das modalidades apresenta resultados que mensuram o ensino superior sob determinado aspecto, entrementes, a visão panorâmica necessária à compreensão global da situação educacional em nível superior é ainda fonte de expectativa.

O estudo, portanto, buscou elucidar as principais contribuições de uma comunidade de prática no processo de auto avaliação de uma instituição de educação superior privada catarinense. Nesse caso, a CoP se constituiu em função da complexidade do processo de auto avaliação e da quantidade de conhecimento crítico que envolve o trabalho da avaliação interna da instituição. Entre os principais produtos e resultados desse envolvimento surgiram projetos, planos, processos e outros mecanismos voltados à orientação do desenvolvimento das pessoas, dos processos, das tecnologias envolvidas e, principalmente, da cultura da instituição.

No modelo de organização em estudo foi possível perceber que a CoP que se criou dentro da IES foi um mecanismo de prática social que visou a modificação da forma de avaliação da instituição. Por sua vez, por meio da proposta instituída para sua criação, nortearam-se os trabalhos e as discussões relacionadas às principais etapas da avaliação, com a aplicação de dinâmicas que auxiliaram no processo de explicitação de conhecimento, o qual se consolidou na construção de um projeto de auto avaliação da instituição.

Por oportuno, outro aspecto a ser destacado refere-se à constituição da CoP com o objetivo de favorecer as discussões acerca de métodos e alinhamento das atividades de preparação, desenvolvimento e consolidação das atividades de auto avaliação. Dessa forma, a CoP passou a promover alguns resultados ao longo de sua construção, tais como: 1) O envolvimento da $\mathrm{CoP}$ na preparação da auto avaliação: em que houve a percepção de que a CoP, definitivamente, assumia o papel da CPA e, simultaneamente, fortalecia a coesão do grupo. Foi promovido o desenvolvimento sistemático de um ambiente informal inserindo a 
cultura de construção, utilização e resgate de conhecimento em um contexto repleto de complexidade. 2) As contribuições da CoP no desenvolvimento da auto avaliação: na qual foi favorecida a escolha da melhor linguagem e de uma abordagem customizada e diretamente relacionada com as expectativas de cada segmento da comunidade acadêmica, propiciando uma maior credibilidade e compreensão de toda a comunidade acadêmica, fortalecendo o processo de auto avaliação institucional; 3) As reflexões da CoP na consolidação da auto avaliação: em que ocasionou o envolvimento de cada um dos membros no processo e permitiu que a cultura de avaliação fosse fortalecer no contexto da instituição, sendo reconhecida pelos gestores.

A guisa de conclusão cumpre salientar que com a participação de $100 \%$ do staff da instituição foi possível identificar que, de fato, a avaliação passava a fazer parte da cultura da comunidade acadêmica. Todavia, embora limitações inerentes a processos de mudança cultural, a importância da retomada da CoP na instituição estudada deve ser ressaltada, haja vista que a continuidade desta ferramenta de difusão do conhecimento foi afetada com o desligamento de três integrantes fundamentais à sua estruturação, impactando os resultados obtidos e o planejamento organizacional voltado às melhorias educacionais conforme preceitos legais de avaliação na área e gerenciamento do conhecimento crítico.

\section{REFERÊNCIAS}

AUGUSTO, Rosana; BALZAN, Newton Cesar. A vez e a voz dos coordenadores das CPAs das IES de campinas que integram o SINAES. Avaliação, Campinas; Sorocaba, SP, v. 12, n. 4, p. 597-622, dez. 2007.

APOSTOLOU, D.; MENTZAS, G., Towards a Holistic Knowledge Leveraging

Infrastructure: the KNOWNET Approach.Proceedings of the 2nd International Conference on Practical Aspects of Knowledge Management. Basel, Switzerland, 29-30, 1998.

ASIAN PRODUCTIVITY ORGANIZATION (APO). Knowledge Management Tools and Techniques Manual. Tokyo: Asian Productivity Organization, 2010.

BARREYRO, G. B.; ROTHEN, J.C. 'SINAES' contraditórios:considerações sobre a elaboração e implantação do Sistema Nacional de Avaliação da Educação Superior.

BOISOT, M. Knowledge assets. Oxford: Oxford University Press, 1998.

BRASIL. Constituição Federal Brasileira. Brasília, 1988. SINAES - Sistema Nacional de Avaliação da Educação Superior: da concepção à regulamentação. $4^{\mathrm{a}}$. ed., ampl. - Brasília : Instituto Nacional de Estudos e Pesquisas, 2007. 
2006.

Decreto 5773 de 9 de maio de 2006. Subchefia para assuntos jurídicos. Brasília

Portaria Normativa $N^{\circ} 40$, de 13 de dezembro de 2007. Institui o e-MEC, sistema eletrônico de fluxo de trabalho e gerenciamento de informações relativas aos processos de regulação da educação superior no sistema federal de educação. Acesso em 10/05/2011. Disponível em: http://www.consuni.ufrj.br/legislacao/portarianormativa40.pdf. Casa Civil. 2007.

. Lei n ${ }^{\circ}$ 10.172/2001 - Dispõe sobre o Plano Nacional de Educação (PNE). Diário Oficial da União, Brasília, 2001.

. Lei n ${ }^{\circ} 10.861 / 2004$ - Dispõe sobre o Sistema Nacional de Avaliação do Ensino Superior (SINAES). Diário Oficial da União, Brasília, 2004.

. Lei no 9.394/1996 - Dispõe sobre as Diretrizes e Bases da Educação (LDB). Diário Oficial da União, Brasília, 1996.

BRINKLEY, Ian. Defining the knowledge economy. Knowledge economy program report. London: The Work Foundation, 2006.

COVAC, José Roberto. Panorama atual da legislação e regulamentação do ensino superior. IN: Seminário Catarinense de Educação Superior. Associação de Mantenedores Particulares do estado de Santa Catarina (AMPESC). Florianópolis. 2011.

CHAPMAN, R.; HYLAND, P. Complexity and learning behaviors in product innovation, Technovation, v. 24 n. 7, p. 553-61, 2004.

CHOO, C. The knowing organization. New York: Oxford University Press, 1998.

COHEN, D.; PRUSAK, L. In good company. How Social Capital Makes Organizations Work. Boston: Harvard Business School Press, 2001.

CRESWELL, J. W. Projeto de pesquisa: métodos qualitativo, quantitativo e misto. 3. ed. Porto Alegre: Bookman, 2010.

DALKIR, Kimiz. Knowledge Management in Theory and Practice. Boston: Elsevier, 2005.

DAVENPORT, T.H.; PRUSAK, L. Conhecimento empresarial.Rio de Janeiro: Campus, 1999.

DU PLESSIS, M. The Role of Knowledge Management in Innovation. Journal of Knowledge Management, v. 11, n. 04, p. 20-29, 2007.

DUBOIS, Nancy; WILKERSON, Tricia. Knowledge Management: Background Paper for the Development of a Knowledge Management Strategy for Public Health in Canada. National Collaborating Centre for Methods and Tools - School of Nursing, McMaster University. Hamilton, 2008. 
DUGUID, P.; BROWN, J. S. Organizational Learning and Communities of Practice: toward a unified view of working, learning, and innovation. Organization Science, v. 2, n. 1, 1991.

FRANCISCO, Thiago H. A.; MELO, Pedro Antônio; MICHELS, Expedito. Reflexões sobre a Avaliação da Educação Superior: As relações entre IGC e PDI. Capivari de Baixo: Editora FUCAP, 2013.

GARCIA, Mauricio. Gestão Profissional em Instituições Privadas de Ensino Superior: um "guia de sobrevivência" para mantenedores, acionistas, reitores, pró-reitores, diretores, coordenadores, gerentes e outros gestores institucionais. Brasil: Hoper, 2006.

HANDZIC, M. et al. Auditing knowledge management practices: model and application.Knowledge Management Research \& Practice, v. 6, p. 90-99, 2008.

IES A. Criciúma. Reunião de constituição do grupo de debates sobre auto avaliação.Livro 01, Num. 1. Pág 5-7. 2008.

. Criciúma. $4^{\mathrm{a}}$ Reunião do grupo de debates sobre auto avaliação.Livro 01, Num. 1. Pág 22-26. 2008.

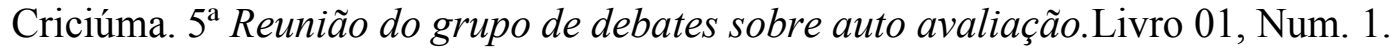
Pág 30-32. 2008.

INEP. Sistema nacional de avaliação da educação superior: da concepção à regulamentação. 3ed. revisada e ampliada - Brasília: Editora INEP, 2007. . 5ed. revisada e ampliada - Brasília: Editora INEP, 2009.

JOINT COMMITTEE ON STANDARDS FOR EDUCATIONAL EVALUATION. The program evaluation standards: a guide for evaluators and evaluation users: a guide for evaluators and evaluation users. 3rd. ed. Thousands Oaks, CA: Sage, 2011.

JYOTI, J.; GUPTA, P.; KOTWL, S. Impact of Knowledge Management Practices on Innovative Capacity: A Study of Telecommunication Sector.The Journal of Business Perspective,p. 315-330, 2011.

LESSER, E. L.; STORCK, J. Communities of Practice and Organizational Performace. IBM System Journal, v. 40, n. 4, 2001.

MILES, Matthew B.; HUBERMAN, Michel A. Qualitative data analysis. An expanded sourcebook. SAGE Publications. 1994.

MORGAN, Gareth. Paradigms, metaphors, and puzzle solving in organization theory.Administrative Science Quarterly. Vol. 25. No 4. 1980.

NICKOLS, Fred. Communities of Practice: what's it like inside? Distance Consulting, 2003. 


\section{CONTRIBUIÇÃO DAS COMUNIDADES DE PRÁTICA PARA O PROCESSO DE AUTOAVALIAÇÃO EM \\ UMA INSTITUIÇÃO DE EDUCAÇÃO SUPERIOR DO SEGMENTO PRIVADO \\ DOI: http://dx.doi.org/10.5007/1983-4535.2016v9n1p97}

NONAKA, I.; TAKEUCHI, H., The knowledge-creating company: how Japanese companies create the dynamics of innovation. University Press, Oxford, 1995.

OCDE. Manual de Oslo - Diretrizes para a coleta e interpretação de dados sobre Inovação. $3^{\mathrm{a}}$ ed., Tradução FINEP, 2007, Disponível em: www.finep.org.br.

RIBEIRO, Célia Maria, COSTA, Eula Maria. Avaliação Institucional: o desenvolvimento humano enquanto percurso e destino. 2002.

RISTOFF, Dilvo. Construindo outra educação:tendências e desafios da educação brasileira. Editora Insular. Florianópolis. 2011.

SANTOS, N. Gestão do Conhecimento Organizacional. Apostila criada para o Programa de Pós-Graduação em Engenharia e Gestão do Conhecimento. Florianópolis, 2005.

SANTOS, N; VARVAKIS, G. Slides utilizados para a aula 2 da disciplina Fundamentos de Gestão do Conhecimento do Programa de Pós-Graduação em Engenharia e Gestão do Conhecimento da Universidade Federal de Santa Catarina. 2014.

SILVA, Assis Leão; ALFREDO, Macedo Gomes. Avaliação institucional no contexto do SINAES: A CPA em questão. Avaliação, Campinas; Sorocaba, SP, v. 16, n. 3, p. 573-601, nov. 2011.

SOMMERMAN, Américo. Inter ou transdisciplinaridade? Da fragmentação disciplinar ao novo diálogo entre os saberes. São Paulo: Paulus. Coleção questões fundamentais da educação. 2006.

SOUZA, Antonio C.; FIALHO, Francisco. A. P.; OTANI, Nilo. TCC Métodos e Ténicas. $1^{\mathrm{a}}$ Ed. Florianópolis: Visualbooks, 2007.

SCHOMMER, Paula Chies. Comunidades de prática e articulação de saberes na relação entre Universidade e Sociedade. Tese apresentada à Escola de Administração de Empresas de São Paulo da Fundação Getulio Vargas, como requisito para obtenção do título de Doutora em Administração de Empresas. São Paulo. 2005.

STALLIVIERI, Luciane. O sistema de ensino superior do Brasil: características, tendências e perspectivas. Universidade de Caxias do Sul, 2006.

SVEIBY, K. E. A nova riqueza das organizações:gerenciando e avaliando patrimônios do conhecimento. Rio de Janeiro: Campus, 1998.

TAYLOR, Steven J.; BOGDAN, Robert. Introduction to qualitative research methods: the search for meanings. John Wiley \& Sons.2. ed. 1984.

URIARTE JR., Filemon A. Introduction to Knowledge Management. ASEAN Foundation, Jakarta, Indonesia, 2008. 
Von KROGH, G.; ROOS, J. Organizational epistemology. New York: St. Martin's Press, 1995.

YIN, Robert R.Estudo caso:planejamento e métodos.2. ed. Porto Alegre: Bookman, 2001.

WENGER, E.Communities of Practice:Learning, meaning and identity.Cambridge, UK: Cambridge University Press, 1998.

WENGER, E.; MCDERMOTT, R.; SNYDER, W. M. Seven principles for cultivating communities of practice.(2002). Disponível em: <http://hbswk.hbs.edu/archive/2855.html $>$ Acesso em: 29 mai. 2014.

WENGER, E.; SNYDER, W. M.Communities of Practice: The Organizational Frontier.Harvard Business Review, jan./fev., p. 139- 145, 2000.

WENGER, E. Communities of Practice and Social Learning Systems. Journal Organization, v.7, n. 2, p. 225-246, 2009.

WENGER, E. Communities of Practice: a brief introduction, 2011. Disponível em https://scholarsbank.uoregon.edu/xmlui/bitstream/handle/1794/11736/A\%20brief\%20introduc tion\%20to\%20CoP.pdf? sequence=1. Acesso em 03 junho de 2014.

WIIG, Karl M.Knowledge Management Foundations: Thinking about Thinking-How People and Organizations Create, Represent and Use Knowledge. Arlington, TX: Schema Press, 1993.

ZAPELINI, Marcelo.B; ZAPELINI, Silvia.M.K.C. Metodologia cientifica e da pesquisa para o curso de Administração. Apostila do curso de Administração. Faculdade Energia de Administração e Negócios, 2007. 